\title{
Antinuclear Antibodies in Children
}
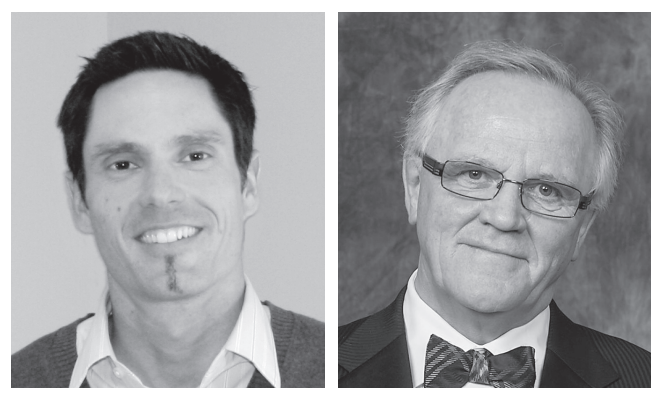

In this issue of The Journal, Sperotto, et al report on a longitudinal study of antinuclear antibodies (ANA) and chronic noninflammatory musculoskeletal pain (CNI-MSP) in schoolchildren ${ }^{1}$. Of 261 subjects, 32 children (12.3\%) were included based on a positive ANA and 77 (29.5\%) based on the presence of CNI-MSP. The positive ANA results were relatively equally distributed with respect to sex and pubertal status. In the ANA-positive cohort, the vast majority of subjects (92.9\%) remained ANA-positive during followup and showed increased titers, but a significant association between ANA positivity and CNI-MSP was not found. In the children with CNI-MSP, the ANA prevalence increased after puberty from $13.4 \%$ to $44.8 \%$ but the increase was not associated with persistence of symptoms or the development of an autoantibody-associated rheumatic disease (AARD). These observations confirmed the results of a previous study showing a lack of a significant association between ANA positivity and CNI-MSP ${ }^{2}$. In addition, a family history of autoimmune diseases did not have a statistically significant influence on the prevalence of ANA positivity during this period. To fully understand and translate these findings into clinical practice, several aspects should be taken into consideration.

\section{Autoantibodies as Precursors or Predictors of AARD}

It has been established that certain autoantibodies, especially ANA, predict the development of AARD ${ }^{3}$. Therefore, a positive ANA without concordant clinical manifestations might be considered a "false-positive" result, but in time it might be a clinically valid result. Therefore, before dismissing an ANA as clinically irrelevant, the specificity of the ANA should be considered and a plan for regular followup considered. In this context, it is important to determine and understand the likelihood ratios (LR) as a measure of post-test probability of disease ${ }^{4}$. Additionally, studies to date on the predictive value of ANA have been based primarily on adults ${ }^{3}$, and similar studies should be replicated in children.
Although the ANA HEp-2 indirect immunofluorescent (IIF) test has been recommended as the method of choice $^{5,6}$, based on the low specificity of a positive ANA in children, its clinical utility is limited in situations with a low pretest probability. Alternative methods, including solid-phase assays (SPA) with defined antigens have similar sensitivities compared to the ANA IIF but offer higher specificity and therefore might have higher value in those cohorts ${ }^{7}$. Consequently, in settings with a low pretest probability, an ANA SPA might provide higher predictive values. Along those lines, studies are needed to compare the clinical utility of ANA IIF and ANA by SPA in children.

\section{Cutoff of IIF Assays}

The determination of the appropriate screening dilution and cutoff for ANA as detected by IIF is also a challenge ${ }^{2}$. For various autoantibody assay kits, the manufacturer cutoffs tend to differ but are widely accepted as being consistent with the performance of the particular assay. However, for ANA IIF on HEp-2 cell substrates, it is assumed that all commercial kits should have the same cutoff value despite differences in growth conditions of the cells, fixations, and other "trade secrets" that are part of the manufacturing process. One cogent and contemporary example of intermanufacturer variation of ANA results is the detection of the novel "rods and rings" IIF pattern, which is restricted to only 1 or $2 \mathrm{HEp}-2$ kits from different commercial sources $^{8}$. Clearly, a comparable clinical performance among kits from different manufacturers is highly desirable, but has yet to achieve a high level of standardization ${ }^{9,10}$.

Another common perspective is that a lower screening dilution should be used for ANA IIF testing in children. However, in Sperotto, et al, $12 \%$ of children were ANA-positive (at 1:80) without any evidence of an AARD. This suggests that the screening dilution used for ANA testing in children requires reconsideration ${ }^{11}$.

See Antinuclear antibodies in schoolchildren, page 1405

Personal non-commercial use only. The Journal of Rheumatology Copyright $@$ ( 2014. All rights reserved. 


\section{ANA Patterns and Anti-DFS70 Antibodies}

Anti-dense fine speckled antigen 70 (anti-DFS70) antibodies, creating a distinctive ANA IIF pattern on HEp-2 cells (Figure 1), is frequently observed in ANA-positive individuals who have no evidence of AARD, although they may be found in many different atopic and inflammatory conditions, and even in apparently healthy individuals ${ }^{12,13,14}$ and in children with autoimmune fatigue syndrome (reviewed in ${ }^{13}$ ).

In the study by Sperotto ${ }^{1}$, the ANA pattern with highest frequency was a fine-speckled pattern. Although a precise definition of the nuclear fine speckled pattern was missing, it is conceivable that some children in this cohort may actually have anti-DFS70 autoantibodies ${ }^{13}$, particularly in light of the failure to find evidence of AARD in these children. The primary target of antibodies associated with the DFS pattern is a $70 \mathrm{kDa}$ protein once referred to as lens epithelium derived growth factor ${ }^{13}$. Until today, no study investigating the prevalence of anti-DFS70 antibodies in pediatric cohorts with a variety of conditions has been published. Therefore, such studies are needed to better understand the prevalence and relevance of these antibodies in children.

A recent report described an 8-year-old female who presented with generalized edema, hypertension, hepatomegaly, and a history of pharyngitis that occurred 3 weeks earlier ${ }^{15}$. Laboratory analyses showed low complement C3 $(6 \mathrm{mg} / \mathrm{dl})$, microhematuria and proteinuria leading to diagnosis of acute glomerulonephritis. A highly positive $(1: 640)$ IIF ANA test with a DFS pattern was found in the absence of anti-dsDNA, anti-extractable nuclear antigens, or antineutrophil cytoplasmic antibodies, which are typically associated with an AARD or systemic vasculitis. However, the presence of anti-DFS70 antibodies was confirmed (by QUANTA Flash DFS70, Inova Diagnostics Inc.) and finally, Streptococcus pyogenes was identified in a throat swab. This report may give some insight into the importance of isolated anti-DFS70 antibodies and AARD-related autoantibodies to improve the LR in a diagnostic setting. Because infectious diseases have yet to be clearly associated with the anti-DFS70 response, careful and systematic studies are needed to explore this possibility.

Consequently, isolated anti-DFS70 antibodies may be useful to exclude AARD in children with positive ANA and a clinical picture that could also be attributable to AARD. This can prevent unnecessary, costly, and invasive investigations and most importantly, inaccurate diagnoses and even inappropriate treatment, which could be attended by significant morbidity.

Sperotto, et $a l^{l}$ demonstrate that ANA are present in significant subsets of children and that titers increase across puberty, but are not associated with the presence or development of AARD. This is in agreement with a previous report indicating that ANA testing in individuals with nonspecific symptoms has limited predictive value ${ }^{16}$. This leads to an obvious conflict because it is important to identify AARD as early and as accurately as possible, to have a window of opportunity for clinical followup and early intervention. As demonstrated in a recently published triage study, testing for anti-ENA and anti-DFS70 antibodies might help to discriminate between patients with a high likelihood ratio of AARD from those who do not have an AARD ${ }^{17}$

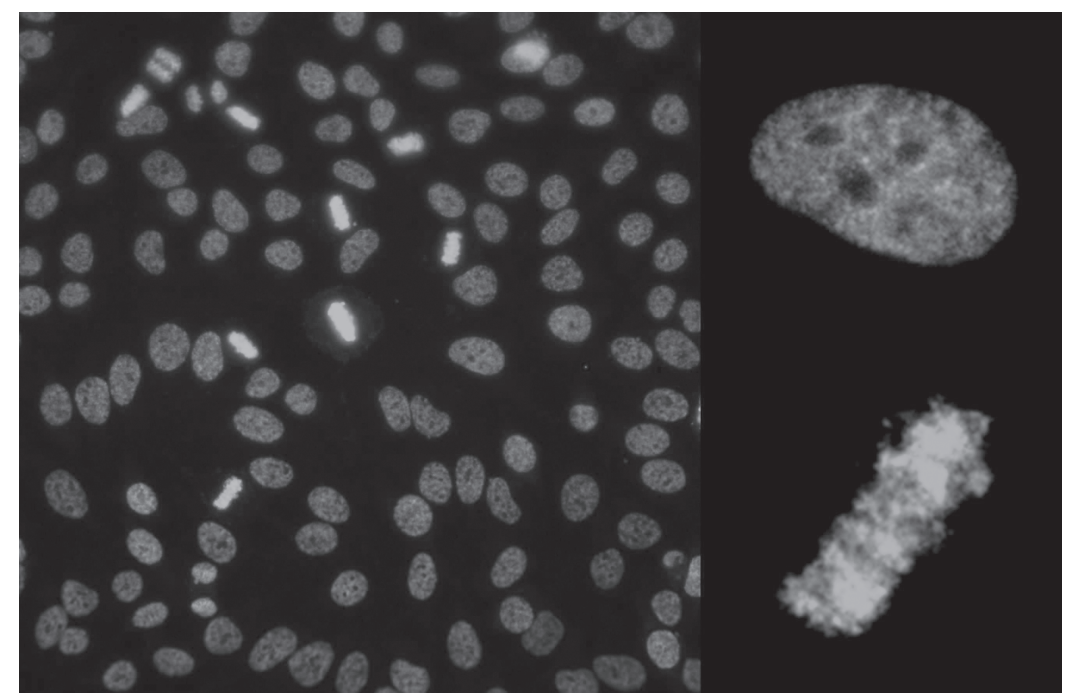

Figure 1. Indirect immunofluorescence pattern generated by antibodies to dense fine speckled antigen 70 (DFS70). The DFS pattern is characterized by the dense fine speckled pattern of interphase cells and the speckled staining of mitotic chromatin. From J Rheumatol 2012;39:2104-10.

Personal non-commercial use only. The Journal of Rheumatology Copyright $\subset$ 92014 . All rights reserved. 
MICHAEL MAHLER, PhD

Department of Research, INOVA Diagnostics Inc.

San Diego, California, USA;

MARVIN J. FRITZLER, MD, $\mathrm{PhD}$,

Department of Medicine,

University of Calgary,

Calgary, Alberta, Canada.

M. Fritzler is a paid consultant, has received honoraria, or has received gifts in kind from ImmunoConcepts Inc. (Sacramento, California), Bio-Rad (Hercules, California), and INOVA Diagnostics (San Diego, California), Mikrogen GmbH (Neuried, Germany), Euroimmun GmbH (Lubeck, Germany), and Dr. Fooke Laboratorien GmbH (Neuss, Germany). M. Mahler is an employee of INOVA Diagnostics Inc., a manufacturer of autoantibody diagnostic kits.

Address correspondence to Dr. Mahler, INOVA Diagnostics Inc., 9900 Old Grove Road, San Diego, California 32131-1638, USA. E-mail:mmahler@inovadx.com or m.mahler.job@web.de

\section{REFERENCES}

1. Sperotto F, Cuffaro G, Brachi S, Seguso M, Zulian F. Prevalence of antinuclear antibodies in schoolchildren during puberty and possible relationship with musculoskeletal pain: A longitudinal study. J Rheumatol 2014;41:xxxx.

2. Tan EM, Feltkamp TE, Smolen JS, Butcher B, Dawkins R, Fritzler MJ, et al. Range of antinuclear antibodies in "healthy" individuals. Arthritis Rheum 1997;40:1601-11.

3. Arbuckle MR, McClain MT, Rubertone MV, Scofield RH, Dennis GJ, James JA, et al. Development of autoantibodies before the clinical onset of systemic lupus erythematosus. N Engl J Med 2003;349:1526-33

4. Bossuyt X. Clinical performance characteristics of a laboratory test. A practical approach in the autoimmune laboratory. Autoimmun Rev 2009;8:543-8.

5. Meroni PL, Schur PH. ANA screening: an old test with new recommendations. Ann Rheum Dis 2010;69:1420-2.

6. Agmon-Levin N, Damoiseaux J, Kallenberg C, Sack U, Witte T, Herold $\mathrm{M}$, et al. International recommendations for the assessment of autoantibodies to cellular antigens referred to as anti-nuclear antibodies. Ann Rheum Dis 2014;73:17-23.

7. Bossuyt X, Fieuws S. Detection of antinuclear antibodies: added value of solid phase assay? Ann Rheum Dis 2014;73:e10.
8. Carcamo WC, Satoh M, Kasahara H, Terada N, Hamazaki T, Chan JY, et al. Induction of cytoplasmic rods and rings structures by inhibition of the CTP and GTP synthetic pathway in mammalian cells. PLoS One 2011;6:e29690.

9. Chan EK, Fritzler MJ, Wiik A, Andrade LE, Reeves WH, Tincani A, et al. AutoAbSC.Org - Autoantibody Standardization Committee in 2006. Autoimmun Rev 2007;6:577-80.

10. Mahler M, Dervieux T. Comments on recent advances and recommendations for the assessment of autoantibodies to cellular antigens referred as antinuclear antibodies. Ann Rheum Dis 2014 Feb 27 (E-pub ahead of print).

11. Perilloux BC, Shetty AK, Leiva LE, Gedalia A. Antinuclear antibody (ANA) and ANA profile tests in children with autoimmune disorders: a retrospective study. Clin Rheumatol 2000;19:200-3

12. Mariz HA, Sato EI, Barbosa SH, Rodrigues SH, Dellavance A, Andrade LE. Pattern on the antinuclear antibody-HEp-2 test is a critical parameter for discriminating antinuclear antibody-positive healthy individuals and patients with autoimmune rheumatic diseases. Arthritis Rheum 2011;63:191-200.

13. Mahler M, Fritzler MJ. The clinical significance of the dense fine speckled immunofluorescence pattern on HEp- 2 cells for the diagnosis of systemic autoimmune diseases. Clin Dev Immunol 2012;2012:494356.

14. Mahler M, Parker T, Peebles CL, Andrade LE, Swart A, Carbone Y, et al. Anti-DFS70/LEDGF antibodies are more prevalent in healthy individuals compared to patients with systemic autoimmune rheumatic diseases. J Rheumatol 2012;39:2104-10.

15. Zago S, Fabris M, Tosolini R, Melli P, Curcio F, Rosolen A, et al. Anti-DFS70 antibodies help to exclude autoimmune pathogenesis in a case of pediatric acute glomerulonephritis with high positive anti-nuclear antibodies [abstract]. International Congress of Autoimmunity, Nice, France; March 26-30, 2014.

16. Abeles AM, Abeles M. The clinical utility of a positive antinuclear antibody test result. Am J Med 2013;126:342-8.

17. Fitch-Rogalsky C, Steber W, Mahler M, Lupton T, Martin L, Barr $\mathrm{SG}$, et al. Clinical and serological features of patients referred through a rheumatology triage system because of positive antinuclear antibodies. PLoS One 2014;9:e93812.

J Rheumatol 2014;41:1260-2; doi:10.3899/jrheum.140480 